\title{
Knowledge Management for Topological Optimization Integration in Additive Manufacturing
}

\author{
Nicolas Gardan \\ CAD/CAE Research Centre DINCCS of MICADO, 08005 Charleville-Mézières, France \\ Correspondence should be addressed to Nicolas Gardan; nicolas.gardan@dinccs.com
}

Received 15 September 2013; Accepted 28 November 2013; Published 17 February 2014

Academic Editors: J.-Y. Hascoet and T. R. Kurfess

Copyright ( 2014 Nicolas Gardan. This is an open access article distributed under the Creative Commons Attribution License, which permits unrestricted use, distribution, and reproduction in any medium, provided the original work is properly cited.

\begin{abstract}
Engineering design optimization of mechanical structures is nowadays essential in the mechanical industry (automotive, aeronautics, etc.). To remain competitive in the globalized world, it is necessary to create and design structures that, in addition to complying specific mechanical performance, should be less expensive. Engineers must then design parts or assemblies that are a better compromise between mechanical and functional performance, weight, manufacturing costs, and so forth. In this context Additive Manufacturing (AM) process offers the possibility to avoid tools and manufacture directly the part. There are numerous technologies which are using different kind of material. For each of these, there are at least two materials: the production material and the support one. Support material is, in most cases, cleaned and becomes a manufacturing residue. Improving the material volume and the global mass of the product is an essential aim surrounding the integration of simulation in additive manufacturing process. Moreover, the layer-by-layer technology of additive manufacturing allows the design of innovative objects, and the use of topological optimization in this context can create a very interesting combination. The purpose of our paper is to present the knowledge management of an AM trade oriented tool which integrated the topological optimization of parts and internal patterns.
\end{abstract}

\section{Introduction}

The optimization of various processes involved in the product life cycle management becomes very crucial. This is the reason why firms have always faced the problem of continuous improvement of their structures in order to predict the optimal forms and structures in the early stages of design. In the research of this compromise, working methods have considerably changed in recent years. Hardware performance, associated with the new methods for solving optimization problems, leads to new methodologies in the design phase.

The optimization of mechanical structures and parts has for a long time been based on a "try/error" methodology. This approach leads to test prototypes whose design is based on the experience of the engineer.

This manual approach is unthinkable today because of the costs and numerous iterations it can cause. The mechanical engineers are required now to integrate the optimization of mechanical structures in their first design phases. This new approach integrates the design requirements and the specifications such as manufacturing constraints, constraints related to business processes, thermomechanical performance, weight requirements, and cost. The engineers can then automatically reproduce, through modeling and numerical optimization software, the work that a designer manually achieved, by adding many advantages [1]:

(i) opportunity to scan a wider design space;

(ii) possibility to implement design of experiments and thus to create approximation functions if needed;

(iii) ability to reach an optimum by using more efficient algorithms.

There are three main categories of shape optimization of mechanical structures [2]: parametric shape optimization, geometric shape optimization, and topological shape optimization. The last one provides, without any explicit or implicit restriction, the best shape possible even if topology changes. It is an appropriate method for the design phase of a new part, because it can explore new concepts and solutions in areas difficult to reach/work for engineers (see a basic example in Figure 1). 

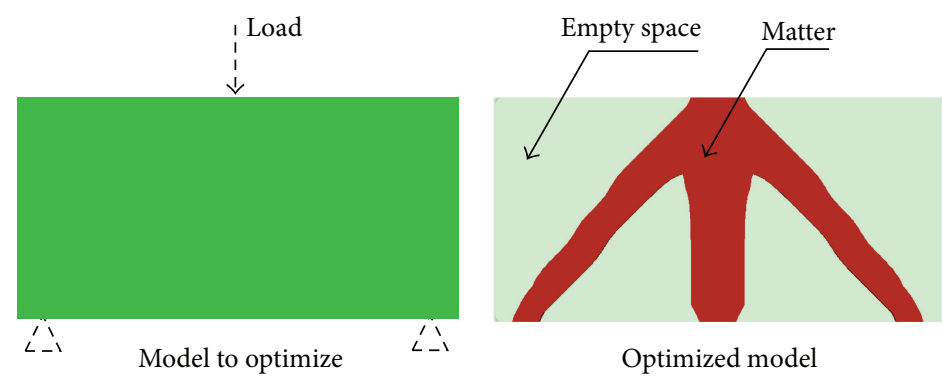

FIGURE 1: Simple example of a topological optimization.

The marriage between additive manufacturing, which can build almost any shape and topology optimization, seems obvious. Indeed, the topology optimization will provide innovative shapes but will require adaptation process from traditional manufacturing (typically a remodeling is required). The objective of this paper is to present the development of a methodology that will serve as a basis for developing a product that will be positioned upstream of the additive manufacturing machine. This software and the associated methodology are intended to be added on all types of additive manufacturing machines. The material and mass saving obtained through the digital optimization can apply for both plastics and metals. In the context of additive manufacturing centre NUM3D, we have access to a selective laser sintering (SLS) machine type, a photopolymerisation machine.... But the approach can be applied to another type of machine; the difference is in the knowledge capitalisation.

The aim of this paper is to present how we manage the knowledge for a good coupling between topological optimization and additive manufacturing. We consider the part and the material support as well (replacement of classic lattice) in our investigation.

\section{Related Works}

AM is nowadays widely used in industrial product development. The main advantage of the additive manufacturing concept used in AM is the ability to create almost any possible shape. This capacity is governed by the built up layer-bylayer process. There are several available technologies based on this additive machining concept [3]: stereolithography, photomasking, selective laser sintering, fused deposition modelling, 3D printing, and so on. Researchers work principally on the influence of part orientation, slicing strategy, and matching internal patterns to improve cost, product quality, built time, and so forth. Numerical topological optimization is a technical break which allows the modelling of really innovative shapes, based on trade knowledge. The union of the two technologies, AM and numerical topological optimization, seems to be very promising and more particularly for steel machining (for a real ROI on mass, e.g.).

The use of optimization in additive manufacturing [4] is generally in the context of the optimization of the build direction [5], parameter optimization trades, optimization construction layers algorithm, and so on. The optimization of the quantity of material used is an important goal. This optimization can match both the product material and the support material. Figure 2 shows the case of using a topology optimization on both the part and the support used (2 optimizations are performed separately. Optimization in the "design" zone is the area that can be optimized and the "nondesign" zone that cannot be changed).

AM machines generally offer the possibility of reducing the mass by using honeycomb shapes, lattices, and so forth. These algorithms model the simplified form without taking into account the specifications of mechanical strength. They are on the majority applied for internal gain of matter. Actually, there are many researches on the influence of cellular structures. Reference [6] has studied the influence of circular and rectangular shapes on the polyamide through compression tests. The study shows the influence of two types of geometric shape based on their use. The circular structure is able to absorb $43.5 \%$ more energy than a rectangular structure that behaves better still in high deformation rates (useful for dynamic fast as crash, explosions, etc.). Reference [7] investigated the use of lattice structures including rapid prototyping to lighten sandwich panels while maintaining their mechanical strength. The study enabled to determine the anisotropy directions of the lattice. The lattice modeling can be adjusted according to the specifications of mechanical strength. Other studies develop specific structures as curved [4], honeycomb [8], cell shape "tetrachirales," [9] or "hexachirales" [10]. However, these studies did not use the opportunity to integrate notion of mechanical strength allowing one to optimize the best shape. The topological optimization through numerical simulation can solve this problem. Reference [11] shows the interest to integrate the topological optimization but also highlights some difficulties such as the following:

(i) the difficulty to manage the drainage system of the part support;

(ii) the size of the CAD file and the difficulty of implementation.

The majority of research mainly focuses on the implementation of new forms (honeycomb lattice) but uses little power topology optimization in numerical simulation which allows one to model the shape automatically. Knowledge management is however necessary to obtain innovative form in the trade context. It is important to note the necessity to 


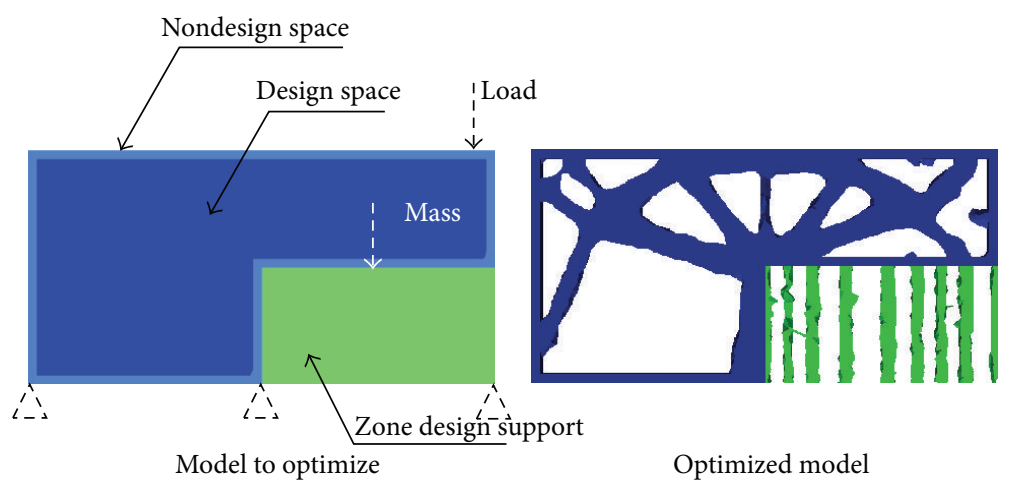

FIGURE 2: Simple example of part and support optimization.

manage the drainage system of the support part. Recently [12] developed a methodology which allows the production of topological optimized part by a low cost FDM. This methodology use the classic optimization process top optimize the mass of the part (included the skin of the part). There is no dedicated optimization tool for AM. Our aim is to develop such a tool. First we have to model knowledge of AM in the topological optimization context.

\section{Methodology and Knowledge Management}

Knowledge management becomes a true stake for the companies. The loss of consciousness due to retirement or turnover and the intrinsic dispersion of knowledge involve high notorious costs. Computer science and in particular artificial intelligence (AI) attempted to provide solutions to help the management of knowledge. AI, while trying in its beginnings to model human intelligence, opened research orientations to set up a decision-making help. The researchers introduce for this purpose the concept of knowledge bases which allow the separation between declarative and procedural statements. The first concrete results appeared with the rules based expert systems, for example, with the software of decision-making medical help MYCIN in 1974 [13]. To reduce production time and production cost, industries are interested in different technologies which depend on research on AI. Knowledge based system (KBS) is born due to AI researches. A KBS is a system which models the knowledge to use it in an application. For example, system expert is a special KBS. There are many researches in using a KBS to CAD modelling. Reference [14] developed an approach for generating assembly drawings from $3 \mathrm{D}$ computer assembly models of mechanical products. This approach uses a specific KBS which saves the strategies of an expert to reuse it. Reference [15] used KBS which includes an expert system to model machine tool. The implemented system uses specific functional features, parametric modeling, and an expert system to modify assembly of a machine tool by applying trade rules. Reference [16] developed a KBS for selection of cutting tools and conditions of turning operations for process planning. The KBS is composed of a base of rules, a base of constraints, a base of facts, and a module called "way of thinking" which represents the logical procedure of an expert for decreasing the solution space. A state of the art on KBS research is done in [17].

3.1. The Knowledge Based System. The knowledge based system (KBS) is dedicated to the AM process and topological optimization. The KBS is using production rules and constraints to represent the declarative knowledge. It is the most usual representation to model heuristic know-how. We are particularly interested in the scenario model [18], that is, the decomposition of a problem in a series of tasks [19]. We base ourselves on the fact that each time an expert resolves a problem, he runs in an intuitive way a scenario. Schank and Abelson [20] set up this structure by affirming that there are thousands of scenarios in the human memory. The different scenarios have been collected by tracking the work of experts.

Our structure is made up of a knowledge base including the following.

(i) A base of scenarios: as we said before experts translate their step of topological optimization and AM process into diagrams of sequences of tasks. They consequently use a scenario of optimization and AM process.

(ii) A base of rules: it contains the production rules and uses the base of constraints for this purpose. These rules are associative, which means that each formalized rules must contain at the same time the context of application of the rule and the condition of the processing of the rule. The rules are rules of production like If (Conditions) Then (Conclusions). The part "Conditions" is the process of the rules and the part "Conclusions" describes the actions to be started in the event of release.

(iii) A base of constraints: it contains all the mechanical laws and the message interaction. The base of constraints contains the set of the constraints having a relationship with the considered field. For example, a constraint can be like Wall_Thickness $>1 \mathrm{~mm}$ to point out the limit of manufacturing to avoid collapsing.

The KBS allows the user to describe the boundary conditions, loads, materials, and so forth. CAD system and CAE system (mesh generator and analysis manager) are fully knowledge 
driven. They are usable by either CAD expert or mechanical engineer and ensure a good usage and the results quality.

Parametric modeling put constraints on geometric model to design modification or to engineering analysis [21]. Constraints can be geometrical (one entity is tangent to another) or knowledge based (e.g., trade rules and constraints). Functional design has to be represented by the vocabulary of the domain. This vocabulary is then used in the definition of trade rules, constraints, and scenarios. Thus, we define specific expression called graphonumerical expression (GNE) and specific parameters called graphonumerical parameters (GNP). The particularity of GNE/GNP is to link the vocabulary to knowledge and to geometry. For example, a sentence like "We have to create at the bottom draft of 20 degrees" is transformed in GNE expression to "Create a the bottom draft of $20^{\circ}$." The GNE expression is saved in a dedicated module in the CAD software (here Rhinoceros3D) which automatically creates functional parameter (GNP). For this example, GNP will be "create (draft) $\left(20^{\circ}\right)$ (\#GeomRef)" (\#GeomRef is a keyword to interact with the CAD user to select geometrical entities).

A GNE contains three classes of different terms. These terms are different according to the context.

Here are the three classes with some examples of terms (several environments included):

(i) action class (create, destroy, modify, consult, remove, apply, to radiate, etc.);

(ii) object class (part, clearance, hole, rib, face, line, edge, point, etc.);

(iii) constraint class (at a tangent to, parallel to, symmetric to, diameter, mass, length, etc.).

A GNE can contain one or several GNP. A GNP is constructed automatically by the graphonumerical engine.

Capitalization has focused around the entire process from design to topological optimization then to AM. Each trade rule affecting the geometry was modelized as EGN. The central idea is to rely the AM parameters to get all information necessary for preparation of the topological optimization and by the way he CAD model. Thus, the knowledge necessary to set data, such as loads, boundary conditions, materials, was modeled to integrate the behavior of the part to the CAD model (Figure 3 ).

3.2. Knowledge Management in AM Process Machining. The aim of the AM knowledge management is to characterize functional parameters for an integration into topological optimization. It is necessary to identify the manufacturing limits to pack the topological optimization solver: it can be the minimum allowed thickness, the possible height without including reinforcement, the dimension of cleaning channels, and so on. We focus, for instance, on the knowledge capitalization on two AM machining processes: SLS and photopolymerization.

Many researches [22-26] present a process of $A M$ machine qualification by manufacturing a test part to quantify defects and grounds. However, these approaches doing not allow pushing to the technological limits of the machine.
This work aims to quantify the defects inherent in each process by the parallel between possible measures in metrology and process-related settings. Our approach is different (and complementary) since we determinate influential parameters and their critical values according to using context, based on [27] research.

The experimental process to recover AM knowledge is based on two types of specimens:

(i) iso campus norm ones manufactured by AM process: their aims are to recover material behaviour (typically elastoplastic law) to use it in simulation (topological optimization). We use specific tools for the measure, for instance, 3D deformation Aramis camera;

(ii) dedicated shapes ones also manufactured by AM process: their aims are to capitalize knowledge management like determining influences parameters: thickness/height limit, pocket depth allowed for powder evacuation, and so on.

We can see on Figure 4 different manufacturing direction and shape of the test parts. Those configurations allow the determination of risk factors.

Our approach involves the study of three very important factors for the topological optimization:

(i) the minimum thickness printable and cleanable without part deterioration: we seek to maximize the minimum thickness of the wire cloth (final material) without loss of geometric and morphological qualities of the part;

(ii) the minimum diameter printable and cleanable without mechanical cleaning: the objective is to size the best channels dimensions for cleaning the internal structure of the piece (allow the powder evacuation);

(iii) the maximum height, in fact the ratio between the projected length and height of the part which may cause a falling down of the matter.

We develop design of experiment (DOE) for different tests: test of the laser temperature impact (for SLS machine), test thickness and height allowed (with cleaning process), test of the manufacturing orientation, test of the plate placement, and so on.

For example, in the case of SLS machine, the tested material is a rapid steel which is metallic powder coat with a polymer. The SLS process involves much knowledge capitalisation because the part is involved in the laser phase, then debinding phase, then infiltration phase, and so on. Before the infiltration phase the part is very breakable and requires specific dimension management. The DOE is based on features analysis. Many features have been studied: holes, slots, ribs, pads, pockets, and so on. They are used in different contexts. For example, the rib is studied in a free way and in an embedded way. We define fourteen tests parts which include this feature (as seen, e.g., in Figure 5) and 3 DOE to drive the experiment. For example, the first eight parts are dedicated to three specific features: vertical holes, slots, free and embedded ribs. The aim is to verify their manufacturability and ability 


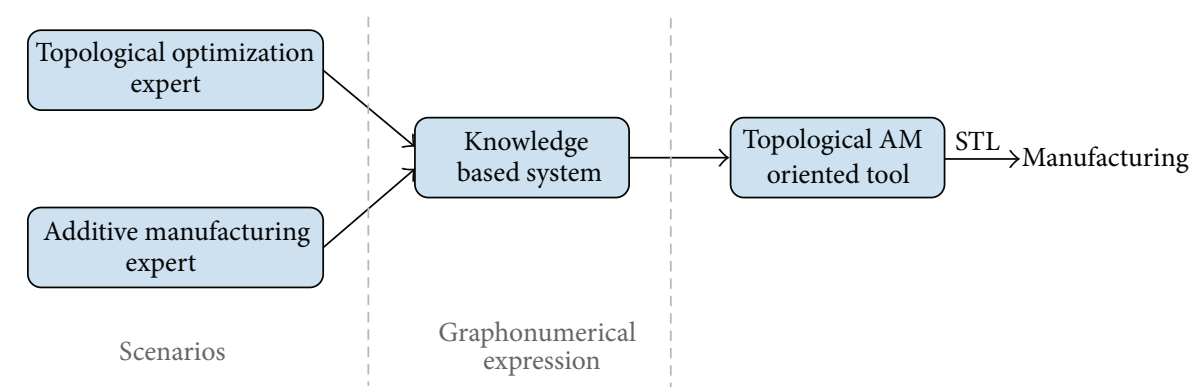

FIGURE 3: Global process.

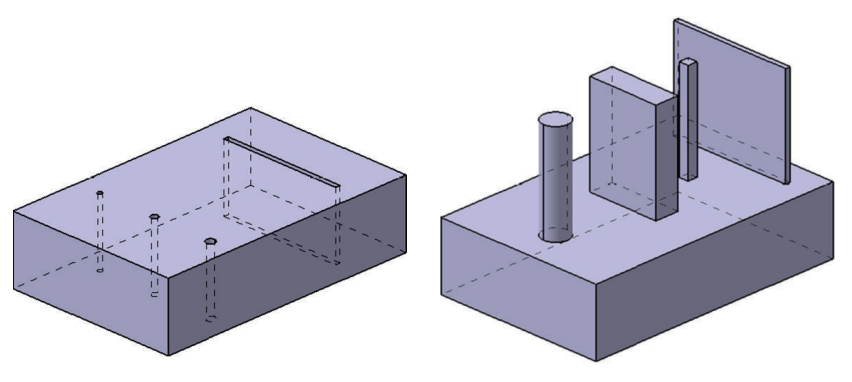

Figure 4: View of the different method of manufacturing.

to be cleaned up. The next algorithm presents a view of the different parameters used in one of the DOE.

[DOE plan 1 - Parts 1-8]

$$
\begin{aligned}
& \text { Hole_Diameter } \mid \text { Slot_Width }=3 \mathrm{~mm} \mid 5 \mathrm{~mm} \\
& \text { Hole_Height } \mid \text { Slot_Height }=7 \mathrm{~mm} \mid 12 \mathrm{~mm} \\
& \text { Part_Height }=15 \mathrm{~mm} \mid 30 \mathrm{~mm} \\
& \text { PartSide_Distance }=5 \mathrm{~mm} \mid 30 \mathrm{~mm} \\
& \text { Laser_Power }=33 \mathrm{~W} \mid 35 \mathrm{~W} \\
& \text { Powder_Temperature }=80^{\circ} \mathrm{C} \mid 85^{\circ} \mathrm{C} \\
& \text { ScanningLaser_Direction }=X \mid Y
\end{aligned}
$$

Table 1 shows the result for free ribs which are used in optimization parameters.

To avoid too many manufacturing tests, we developed an associated numerical simulation test based on the DOE experiment. We used iso campus specimens in tensile testing machine to determine elastoplastic material behaviour.

3.3. Knowledge Management in Topological Optimization. In topological optimization we try to solve a specific structural problem (as seen in (1)):

$$
\min \sum_{i=1}^{n} w_{i}\left(\frac{f_{i}(x)-T_{i}}{T_{i}}\right)^{2} g_{j}(x) \leq 0 \quad j=1, m .
$$

In this equation, $f_{i}(x)$ are structural responses obtained from a classic finite element analysis (FEA) and $f_{i}(x)$ is the constraints function. During the calculation, if $g=$ 0 then constraints are active, if $g<0$ then constraints are considered as inactive, and if $g>0$ constraints are
TABLe 1: Results for rib DOE analysis.

\begin{tabular}{lccc}
\hline Parameter & Operator & Value & Result \\
\hline Thickness & $<$ & $1 \mathrm{~mm}$ & Nonfeasible (matter collapsing) \\
Thickness & $=$ & $2 \mathrm{~mm}$ & Deformation for height $>10 \mathrm{~mm}$ \\
Thickness & $=$ & $3 \mathrm{~mm}$ & Deformation for height $>40 \mathrm{~mm}$ \\
Width & $>$ & $15 \mathrm{~mm}$ & Cleaning constraint
\end{tabular}

To help the global strength, pins can be brought back

defined as violated, the calculation cannot reach the objective as well, defined by the target values $T_{i}$. The values of the weighting factors, $w_{i}$ are chosen by experiment, in order to obtain a suitable rapid and stable convergence of the scheme [28]. In topology optimization design variables are element densities and are managed in our work by SIMP method. As seen before, specific techniques need to be introduced to penalize intermediate densities and to force final design to be represented by densities of 0 or 1 for each element. This particular adjustment is really important for the knowledge management. The penalization technique used is the power law representation of elasticity properties which can be expressed as shown in (2), where $\underline{K}$ is the penalized stiffness matrix, $K$ the real stiffness matrix, $\rho$ the density, and $P$ the penalization factor which has to be superior to 1 :

$$
\underline{K}(\rho)=\rho^{P} K .
$$

This penalized factor will have a very relevant impact on our problem. Indeed, in the case of AM, we have to manage the minimum length which represents the thickness of the different walls. Belonging to the machine process, this value is determined by experiment data. A simple example is shown in Figure 6: on a simple C-CLIP optimization, the penalization factor management allows a bad or a good management of knowledge.

A topology optimization problem relative to an AM process can be defined by the following.

(i) Design spaces: a design space corresponds to the interior of the objects and a nondesign space corresponds to the skin of the object (or any other area that should not be modified such as the apertures for cleaning). These areas are identified in CAD model.

(ii) Design variables: they are the set of parameters of the design space related to the AM process to define 


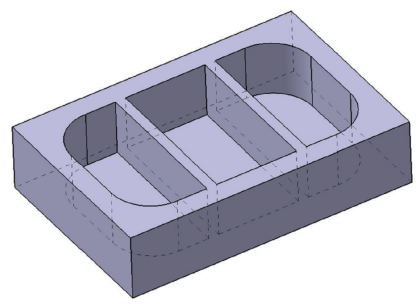

Embedded rib

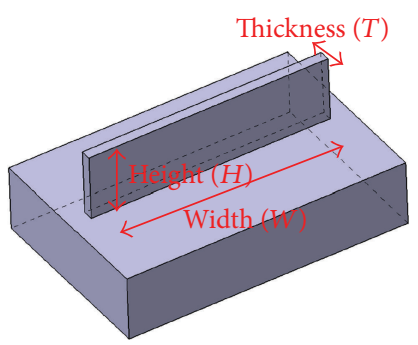

Free rib

FIGURE 5: Two kinds of tested rib features.

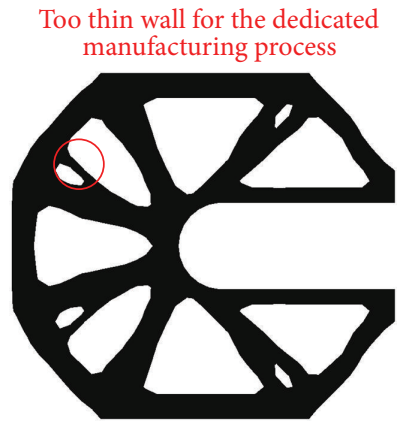

First model

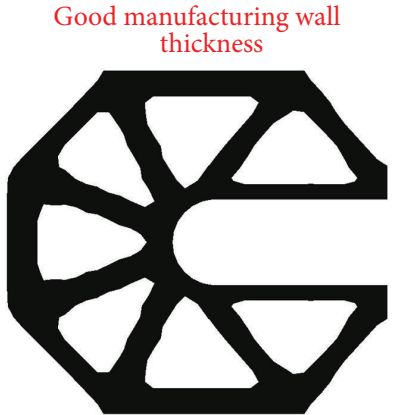

Second model with penalty regulation

Figure 6: Penalization factor management on a C-CLIP example.

the initialization problem of topological optimization. We find here the penalization factor, the pattern repetition, and so on.

(iii) Responses: responses correspond to structural responses, calculated in a finite element analysis, or combinations of these responses to be used as objective and constraint functions in a structural optimization. Available responses could be for example, static displacement, mass, volume, temperature, natural frequency, and so on.

(iv) Constraints: constraints are based on responses by marking them with specific values.

(v) Objective: the objective function is, as we have seen before, the minimisation (or the maximisation) of the problem, here specific responses (e.g., the aim is to manage one response by objective function).

\section{Applications}

To validate our methodology and prepare the software integration, we first verified our assertion with commercial software. We developed in Rhinoceros3D the KBS (in Python) and a basic interface which helps the designer to prepare the CAD model and launch in background Optistruct solver (see Figure 7).

For instance, the topological optimization scenario is limited to one objective: the weight gain.

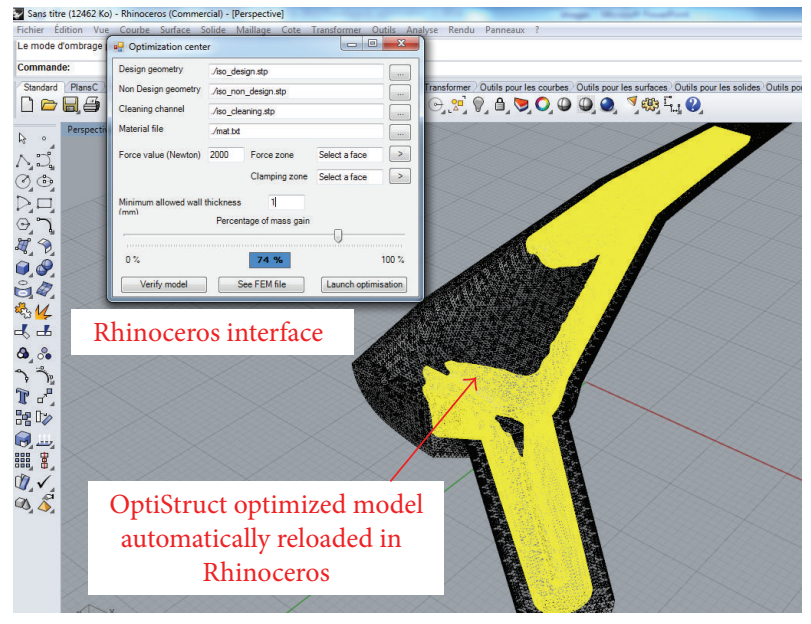

FIGURE 7: View of Rhinoceros program and half of the hip model.

The first step is to define design variables like the penalization factor as we explained before. This penalization factor is defined according to the minimal thickness obtain by test. We define then two specific responses:

(i) compliance response: the compliance is the strain energy of the structure and can be considered a reciprocal measure for the stiffness of the structure. 


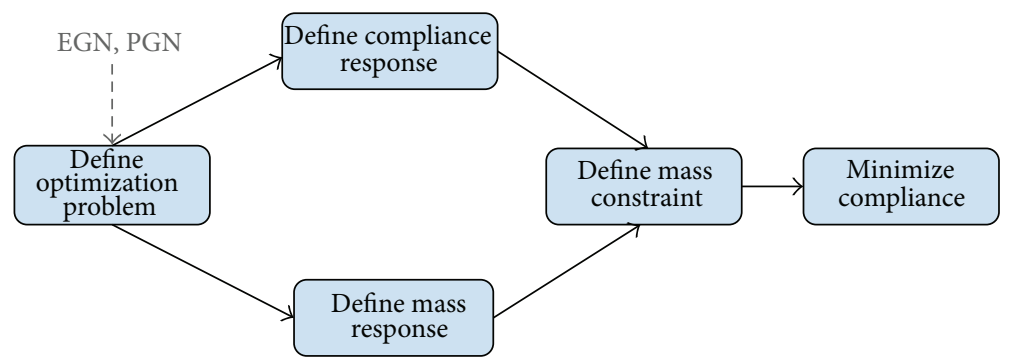

FIgURE 8: Mass scenario for topological optimization in AM.

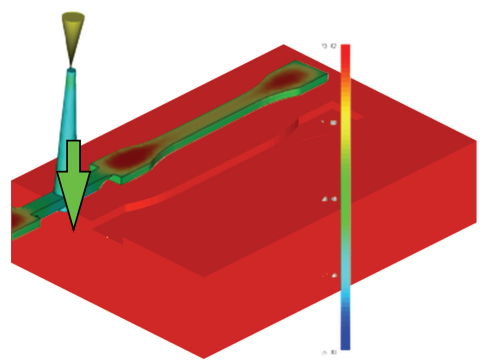

Front

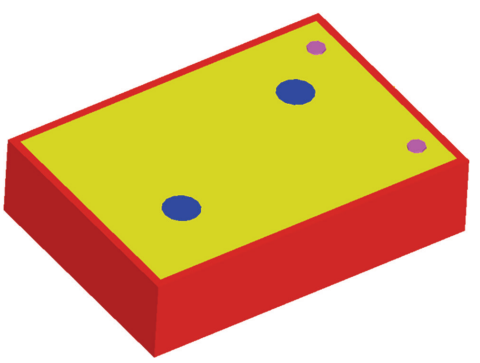

Back

FIGURE 9: View of the insert and the different design spaces.

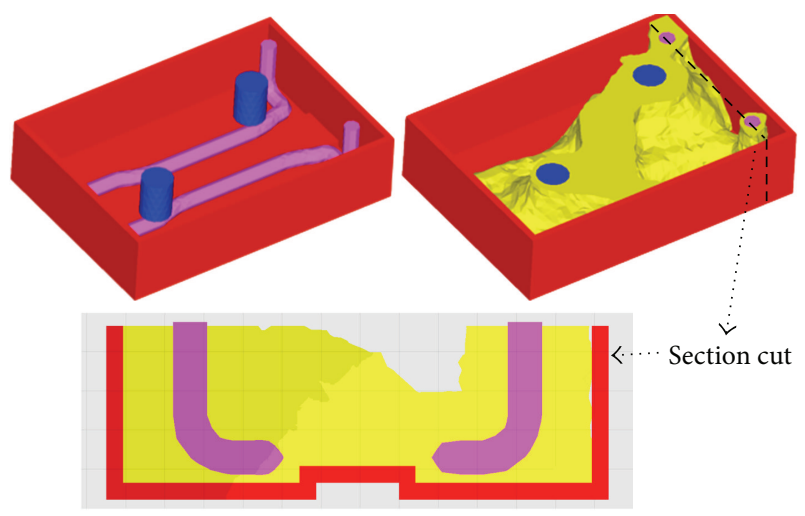

FIgURE 10: Set of the cleaning channels in the numerical model.

A global measure of the displacements is the compliance of the structure under the prescribed boundary conditions.

(ii) fraction of mass response: the fraction mass response is the material fraction of the designable material mass. It corresponds to a global response with values between 0 and 1 . This allows the user to specify intuitive question like "I want to gain $30 \%$ of mass."

The next step is to minimize the compliance. Generally, in optimization, the compliance is used to evaluate the stiffness. Minimizing the compliance means to have a stiffer structure. The lower the compliance, the higher the stiffness of the structure. So, the problem statement involves the objective functional of the strain energy which has to be minimized (see the scenario in Figure 8).
A various number of application has been done to test the different scenarios. We limited our application for instance into to AM process available in the NUM3D platform: a SLS machine and a photopolymerisation machine (resp. DTM and EDEN 260V).

One of our examples is to obtain a significant weight gain on a mold insert for manufacture test specimen. The manufacturing is done on an EDEN $260 \mathrm{~V}$. The objective of weight gain is fixed to $50 \%$ and the insert has to resist to temperature, pressure, and so on of the injection process. The knowledge relative to the EDEN $260 \mathrm{~V}$ and the constraints due to the using of the insert is modeled in the KBS. We can see on Figure 9 the nondesign spaces and the design space (in yellow).

One of the difficulties of this object is to correctly integrate the cooling channel especially for the support cleaning. We see on Figure 10 on the left the cooling channel and on the right the topological result.

Figure 11 shows the manufacturing process of the obtained shape with almost $47 \%$ of weight gain. The difference with the theoretical $50 \%$ target is explained by numerical adaptation like mesh size, material behavior and so on. It is important to note that there is no transformation between the optimized shape and the manufacturing process. We keep the AM principle based on the "one click" manufacturing based on the STL format.

\section{Conclusion}

We present in this paper the knowledge management developed to link topological optimization to additive manufacturing. This coupling is very interesting for many reasons: topological optimization provides very innovative shape 


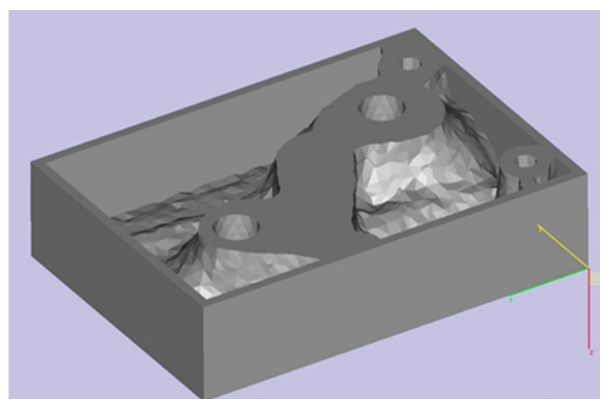

(a)

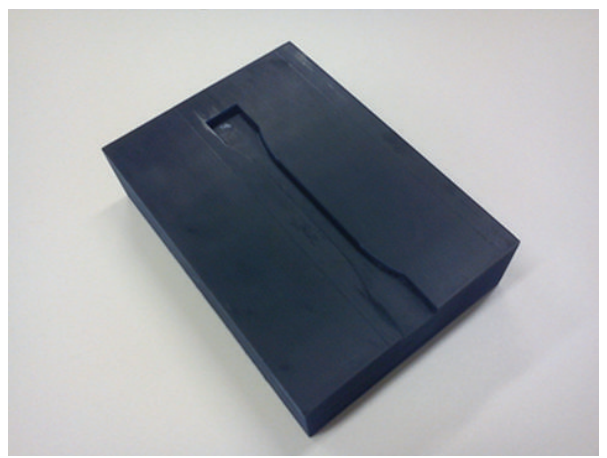

(c)

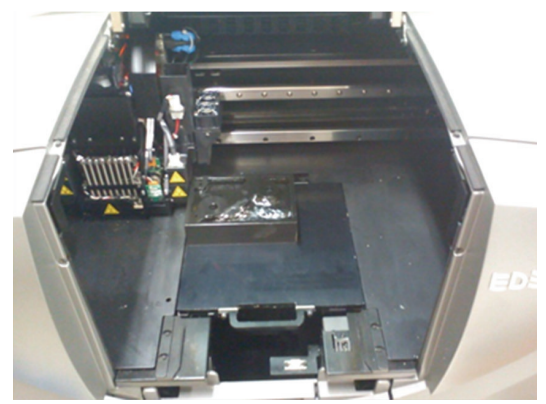

(b)

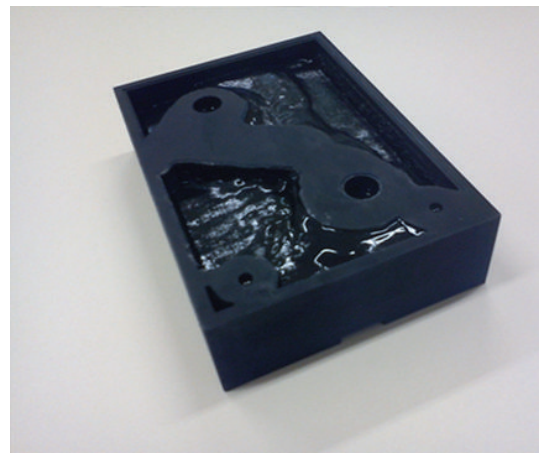

(d)

FIGURE 11: View of manufacturing: (a) numerical model obtained with topological optimization dedicated to AM, (b) view of the manufacturing process, and (c) and (d) view of the manufactured insert.

but sometimes is difficult to manufacture with traditional manufacturing process and additive manufacturing, with the layer-by-layer concept, allows the production of almost any kind of shape.

Our approach is based on the definition of scenarios and the integration of AM knowledge with the help of graphonumerical expression. The demonstrator, developed for instance, in commercial software, allows the optimization in AM context of the part (traditional topological optimization but in AM context) and the interior of the part as well (to replace classic honeycomb lattice). This particularity allows keeping external skin and has a really weight gain by removing matter with full respect to mechanical strength. For instance, we test a dozen of parts which give very good results like a prosthetic implant used in a hip replacement surgical procedure. One of the improvements is to manage automatically the drainage system which has to be included manually, for instance.

Actually the project is currently growing up in a huge project. Many industrial partners are interested in the development of dedicated AM topological optimization tool.

\section{Conflict of Interests}

The author declares that there is no conflict of interests regarding the publication of this paper.

\section{Acknowledgments}

This project is partly based on the platform NUM3D (partners: UTT, ENSAM, and CReSTIC for URCA). The author also thanks the Champagne-Ardenne region and the General Council. Advice on AM material from Julien Gardan of Aztech society and help from Alexandre Schneider from the University of Reims deserve the authors' heartfelt thanks.

\section{References}

[1] G. Allaire, C. Dapogny, and P. Frey, "Topology and geometry optimization of elastic structures by exact deformation of simplicial mesh," Comptes Rendus Mathematique, vol. 349, no. 17-18, pp. 999-1003, 2011.

[2] A. Thakur, A. G. Banerjee, and S. K. Gupta, "A survey of CAD model simplification techniques for physics-based simulation applications," Computer-Aided Design, vol. 41, no. 2, pp. 65-80, 2009.

[3] T. Wohlers, "Additive manufacturing and 3D printing-state of the industry," Wohlers Report: Annual Worldwide Progress Report, 2012.

[4] L. M. Galantucci, F. Lavecchia, and G. Percoco, "Study of compression properties of topologically optimized FDM made structured parts," CIRP Annals, vol. 57, no. 1, pp. 243-246, 2008.

[5] A. M. Phatak and S. S. Pande, "Optimum part orientation in rapid Prototyping using genetic algorithm," Journal of Manufacturing Systems, vol. 31, pp. 395-402, 2012.

[6] M. Vesenjak, L. Krstulović-Opara, Z. Ren, and Ž. Domazet, "Cell shape effect evaluation of polyamide cellular structures," Polymer Testing, vol. 29, no. 8, pp. 991-994, 2010.

[7] Y. Sugimura, "Mechanical response of single-layer tetrahedral trusses under shear loading," Mechanics of Materials, vol. 36, no. 8, pp. 715-721, 2004.

[8] H. Abramovitch, M. Burgard, L. Edery-Azulay et al., "Smart tetrachiral and hexachiral honeycomb: sensing and impact 
detection," Composites Science and Technology, vol. 70, no. 7, pp. 1072-1079, 2010.

[9] W. Miller, C. W. Smith, F. Scarpa, and K. E. Evans, "Flatwise buckling optimization of hexachiral and tetrachiral honeycombs," Composites Science and Technology, vol. 70, no. 7, pp. 1049-1056, 2010.

[10] D. Prall and R. S. Lakes, "Properties of a chiral honeycomb with a Poisson's ratio of-1," International Journal of Mechanical Sciences, vol. 39, no. 3, pp. 305-314, 1997.

[11] P. Rochus, J.-Y. Plesseria, M. van Elsen, J.-P. Kruth, R. Carrus, and T. Dormal, "New applications of Rapid Prototyping and Rapid Manufacturing (RP/RM) technologies for space instrumentation," Acta Astronautica, vol. 61, no. 1-6, pp. 352-359, 2007.

[12] R. Rezaie, M. Badrossamay, A. Ghaie, and H. Moosavi, "Topology optimization for fused deposition modeling process," Procedia CIRP, vol. 6, pp. 521-526, 2013.

[13] E. H. Shortliffe and B. G. Buchanan, Rule-Based Expert Systems: MYCIN Experiments of the Stanford Heuristic Programming Project, Addison-Wesley, Reading, Mass, USA, 1984.

[14] K.-Z. Chen, X.-A. Feng, and L. Ding, "Intelligent approaches for generating assembly drawings from 3-D computer models of mechanical products," Computer-Aided Design, vol. 34, no. 5, pp. 347-355, 2002.

[15] S. Myung and S. Han, "Knowledge-based parametric design of mechanical products based on configuration design method," Expert Systems with Applications, vol. 21, no. 2, pp. 99-107, 2001.

[16] S. C. Park, "Knowledge capturing methodology in process planning," Computer-Aided Design, vol. 35, no. 12, pp. 1109-1117, 2003.

[17] S.-H. Liao, "Expert system methodologies and applicationsa decade review from 1995 to 2004," Expert Systems with Applications, vol. 28, no. 1, pp. 93-103, 2005.

[18] N. Gardan and Y. Gardan, "An application of knowledge based modelling using scripts," Expert Systems with Applications, vol. 25, no. 4, pp. 555-568, 2003.

[19] A. Aamodt and E. Plaza, "Case-based reasoning: foundational issues, methodological variations, and system approaches," $A I$ Communications, vol. 7, no. 1, pp. 39-59, 1994.

[20] R. C. Schank and R. P. Abelson, Scripts, Plans, Goals and Understanding: An Inquiry Into Human Knowledge Structures, Lawrence Erlbaum Associates, Hillsdale, NJ, USA, 1977.

[21] F. Danesi, N. Gardan, Y. Gardan, and M. Reimeringer, "P4LM: a methodology for product lifecycle management," Computers in Industry, vol. 59, no. 2-3, pp. 304-317, 2008.

[22] A. Bernard and A. Fischer, "New trends in rapid product development," CIRP Annals, vol. 51, no. 2, pp. 635-652, 2002.

[23] D. Kochan, C. C. Kai, and D. Zhaohui, "Rapid prototyping issues in the 21st century," Computers in Industry, vol. 39, no. 1, pp. 3$10,1999$.

[24] J.-P. Kruth, M. C. Leu, and T. Nakagawa, "Progress in additive manufacturing and rapid prototyping," CIRP Annals, vol. 47, no. 2, pp. 525-540, 1998.

[25] D. Scaravetti, P. Dubois, and R. Duchamp, "Qualification of rapid prototyping tools: proposition of a procedure and a test part," The International Journal of Advanced Manufacturing Technology, vol. 38, no. 7-8, pp. 683-690, 2008.

[26] T. H. C. Childs and N. P. Juster, "Linear and geometric accuracies from layer manufacturing," CIRP Annals, vol. 43, no. 1, pp. 163-166, 1994.
[27] B. H. Lee, J. Abdullah, and Z. A. Khan, "Optimization of rapid prototyping parameters for production of flexible ABS object," Journal of Materials Processing Technology, vol. 169, no. 1, pp. 5461, 2005.

[28] M. P. Bendsøe and N. Kikuchi, "Generating optimal topologies in structural design using a homogenization method," Computer Methods in Applied Mechanics and Engineering, vol. 71, no. 2, pp. 197-224, 1988. 

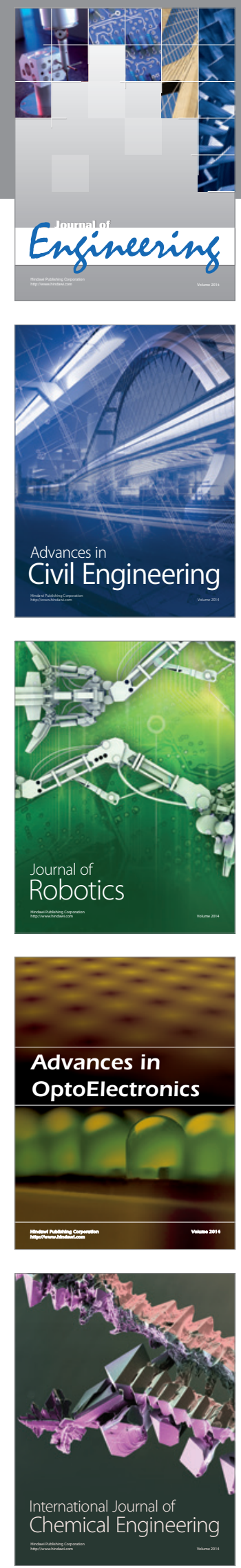

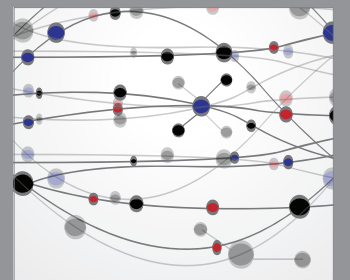

The Scientific World Journal
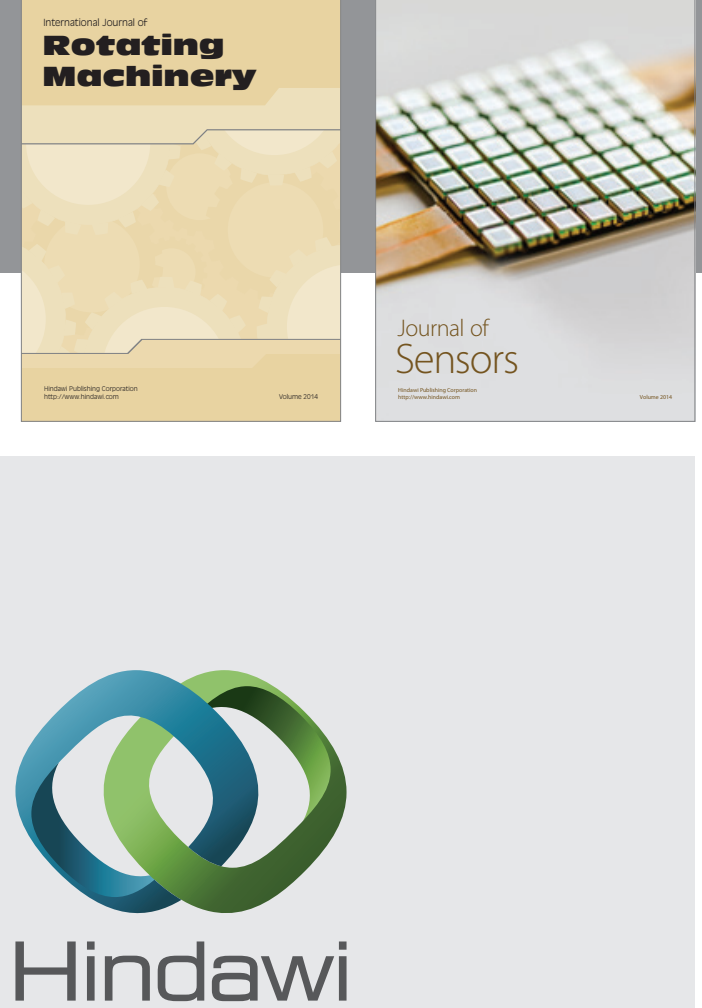

Submit your manuscripts at http://www.hindawi.com
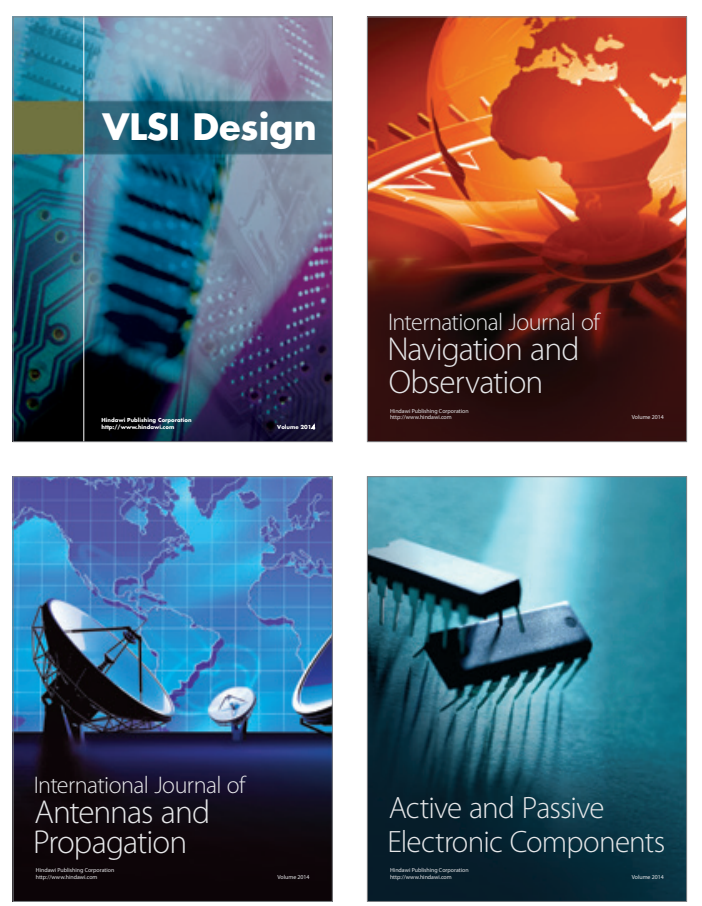
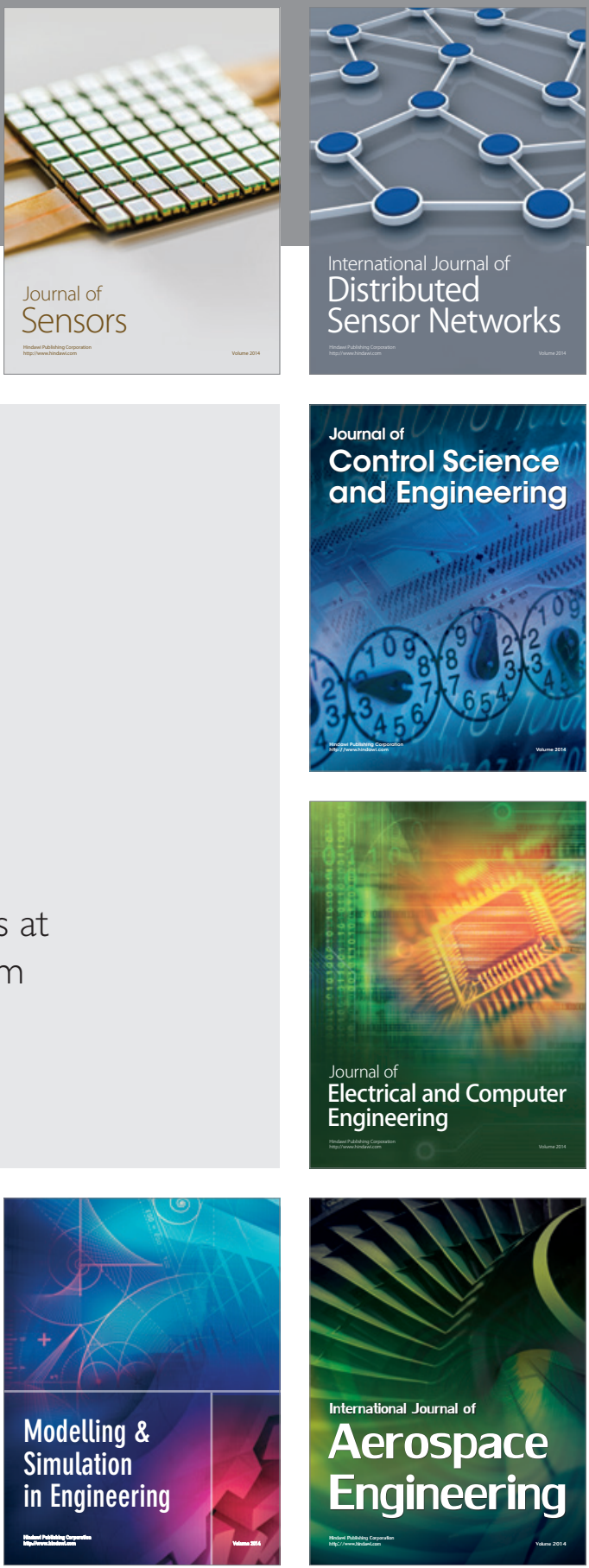

Journal of

Control Science

and Engineering
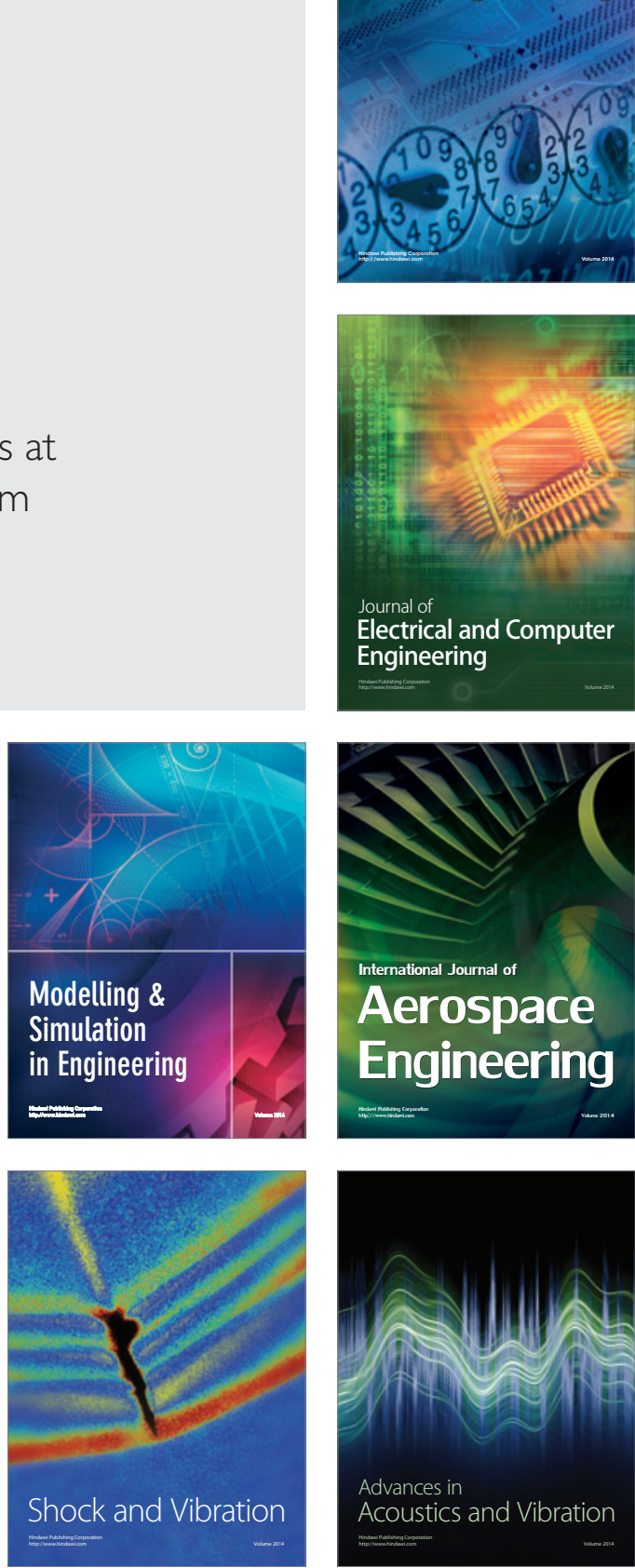